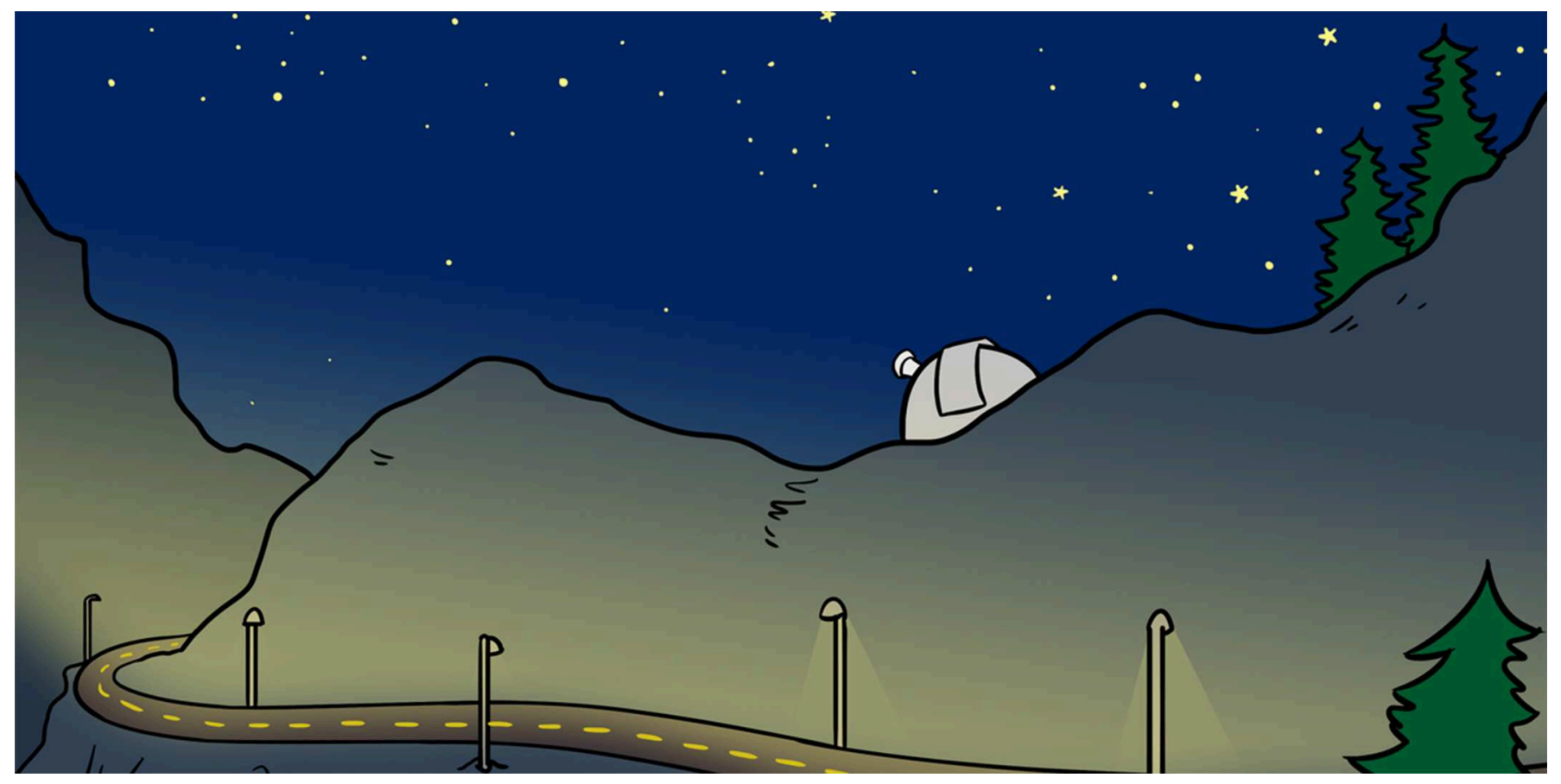

\title{
WHY PUT TELESCOPES AT THE TOP OF MOUNTAINS AND OTHER STRANGE PLACES?
}

\section{Edward Gomez *}

Las Cumbres Observatory Inc., Goleta, CA, United States

YOUNG REVIEWER

GREG

AGE: 15
Telescopes are the fundamental instruments that astronomers use to make discoveries about the Universe. With technological advances over the past 20 years, it is now possible to create networks of robotic telescopes. These robotic telescopes are beginning to revolutionize scientific investigations and also make them accessible to students and teachers, in ways never seen before.

\section{A SHORT HISTORY OF THE TELESCOPE}

During the middle ages, if you were to look up at the night sky you would have seen something very different from the view you see when looking at the sky today. This is partly because the skies are now much brighter because of streetlights and other outdoor lighting, in all but the remotest places. It is also because our modern understanding of the Universe is so totally different from the ancient view of the Universe. 


\section{SUPERNOVA}

The explosion of a massive star at the end of its life.

\section{OBSERVATION}

The job of moving a telescope to a certain part of the sky and then taking pictures.
To ancient people, the stars appeared as fixed points of light, only interrupted by the planets meandering slowly through the night sky. Occasionally there was the flash of a meteor, a short-lived shooting star. Very rarely, a comet might be visible in the sky, and even more rarely, a supernova explosion would suddenly burn brightly in the sky, over the period of months or sometimes years.

Four hundred years ago there was a revolution, as Western Europe started thinking about art and science in a very different way, similar to the thinking of the ancient Greeks. This period was called the Renaissance. During the Renaissance, in the early part of the seventeenth century, the astronomical telescope was "invented" almost simultaneously by Thomas Harriot and Galileo Galilei (the telescope was invented by Hans Lipparshey, but Harriot and Galileo were the first to use it for astronomy).

Science changed forever. Galileo observed Jupiter and found that it had moons that orbited the planet itself and not the Sun or the Earth. He was fascinated with Jupiter and its moons and became convinced that the solar system was similar, with the Sun, not the Earth, as its center. Galileo got in lots of trouble for asserting this. Such a viewpoint was against the official doctrine of the Church, so that might be why Galileo got in trouble, but it also might have been because he was a bit too forceful in expressing his opinions and made powerful enemies. Either way, Galileo found himself under house arrest.

Isaac Newton, famous for his theory of gravity and his laws of motion, was also a pioneer in the theory and use of lenses and mirrors. Newton invented a telescope that used a mirror, instead of just lenses, which has become the foundation for modern optical astronomy. Telescopes with mirrors can be made much larger than ones that use lenses, and the mirrors also make them shorter than refracting or lens-based telescopes.

Gradually, as people turned more and better telescopes on the night sky, our understanding of the Universe expanded. There came a point when the telescope became limited not by its technology, but by its location. The very early telescopes of Galileo, Harriot, and Newton were a huge improvement over naked-eye observations, but as the technology developed to fine-tune the process of constructing a telescope, another problem reared its head.

\section{REMOTE TELESCOPES}

The earth's atmosphere is essential for the survival of humans, because it allows us to breathe, keeps out the cold of space, and shields us from the Sun's harmful radiation, as well as cosmic rays, small meteors and other dangers from space. However, the atmosphere presents many problems for astronomers, because it causes starlight to scintillate, 
Figure 1

Haleakala mountain in Maui, Hawaii. This location is home to many astronomical observatories, including Las Cumbres Observatory's Hawaii node.

\section{OBSERVATORY}

A building that houses a telescope (or telescopes).

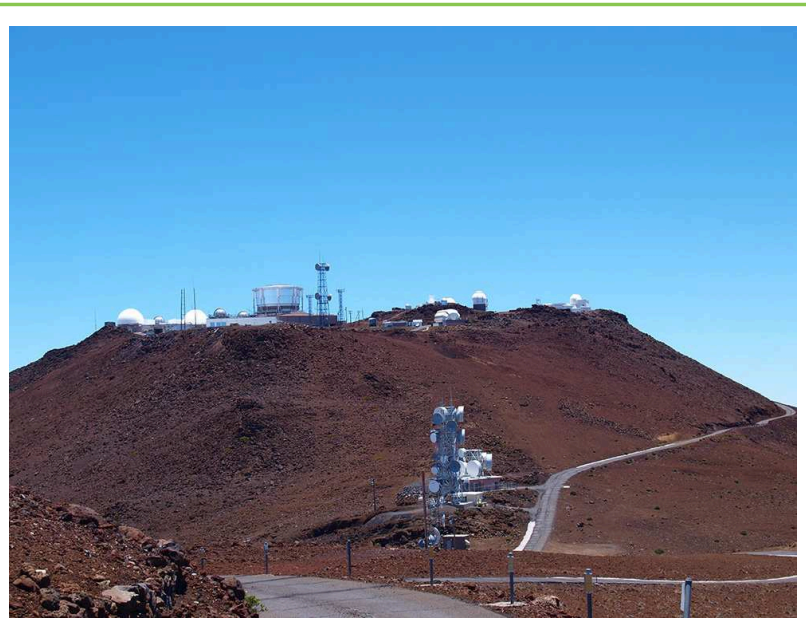

Figure 1

or twinkle. This is a very similar effect to heat haze, and it comes from pockets of the atmosphere continually changing in temperature and density.

Minimizing the effect of the atmosphere on observations using telescopes makes a huge difference to the quality of the observations. Astronomy is a unique science, in that it is almost totally passive. Humans mostly sit on Earth and collect light, because that is almost all we can do to learn about the Universe. To be an astronomer, you have to be resourceful and extract every bit of data from every particle of light from space. Only relatively recently have we been able to travel into space, and the distance we have traveled is microscopic compared with the distances of the objects we were observing with telescopes. We are still a very long way from being able to visit other stars and galaxies to discover more about how they form, or to take measurements of a black hole from close quarters.

The need to minimize interference from the atmosphere and extract as much information as possible from the tiny amount of light available means that we need to put our telescopes in strange, exotic locations, like at the top of mountains or even the summits of dormant volcanoes. These remote locations minimize the twinkling, because the atmosphere is thinner in these spots. If a location is chosen carefully, we can also eliminate the glare from outdoor lights, which reflect off the atmosphere and give the sky an artificial brightness. Air quality is important for good observations, because any particles in the air will reflect light and also make the sky glow. Finally, weather has a substantial impact on the quality of observations. Ideally, observatories should be located as high above the clouds as possible.

Taking all of these factors into consideration, it is a good idea to put an observatory in a remote place, away from cities and built-up areas, a few kilometers above sea level (see Figure 1). 
CHARGE COUPLED DEVICE (CCD)

CCDs are used inside digital cameras to record the light and turn it into digital pictures

\section{TELEMETRY}

Information sent from sensing equipment like telescopes to an (often far away) control center. In the case of robotic telescopes, this can be information about the position of the telescope or measurements from the weather sensors.

\section{SLEW}

The motion of a telescope as it moves into position to observe a target.
Once the telescope is placed in an extremely high-quality location, the challenge becomes using this instrument effectively for scientific investigations. Unlike telescopes that you might purchase to use from your home, very few astronomical observatories have eyepieces attached to their telescopes, and even fewer professional astronomers look through eyepieces to record measurements. Instead, many telescopes that look at visible light use charge coupled devices (CCDs) to capture light. CCDs are the devices inside all digital cameras that detect light particles. Attaching a high-quality digital camera to a telescope gives astronomers a reliable way of precisely measuring the amount of light being transmitted from an astronomical source. By using colored filters with these cameras, astronomers can make measurements of how each astronomical target looks in different colors. Combining this with a knowledge of astrophysics allows astronomers to piece together what the object is and what processes are happening throughout the object's life.

\section{ROBOTIC TELESCOPES}

If you only have one telescope, you are at the mercy of the weather. Many observatories still require astronomers to be on site to operate them, which can be very frustrating if there is bad weather.

With carefully designed systems for monitoring things like the weather, sky conditions, telescope statuses, telescope telemetry, control over all mechanical and electrical aspects of the observatory, and connection of the observatory to the internet, most observatories can be made robotic and remotely operable. These observatories still require a person to have remote control of every aspect of their operation, for example, opening the observatory dome, moving the telescope, and starting a camera exposure. The key, however, is that this person could be anywhere in the world controlling the telescope [1].

\section{AUTOMATED ROBOTIC OBSERVING}

Once an observatory is set up for remote operation, then the really challenging part is to remove the telescope operator and make the observatory capable of "thinking" for itself, in a limited way. This requires sophisticated computer programs that can make decisions about whether the sky conditions are good and what to observe, from a pre-scheduled queue. Back-up plans also have to be built in, in case something goes wrong. If the telescope gets stuck while slewing (moving into position), the observatory's digital brain has to recognize the signs and alert a person so that the telescope will not be damaged and it can be repaired. 
To make an observatory remotely operable will require a lot of effort and cost, but it can make the difference between an average observatory and a world-class, efficient observatory.

Ideally, the observatory's digital brain should also take all the observation requests from astronomers around the world and put them into an efficient schedule, with a minimum number of gaps. This will allow more observations of more astronomical objects for more people, significantly increasing the productivity of the observatory. If the observatory has an online repository of its data, astronomers can observe and then analyze the data within minutes [2].

\section{A NETWORK OF INTELLIGENT ROBOTIC TELESCOPES}

There is still the problem of only having one telescope, in the case of bad weather or an extended period of maintenance. The answer is simple: build more telescopes and build them in different locations. Having telescopes in both the northern and southern hemispheres and spreading them out evenly across the Earth allows for observation of the whole sky. And, even better, adding an internet connection and some special software can create a telescope network that is more powerful than the sum of its parts. Such an observatory becomes global, and its digital brain has far more resources to play with. A network of robotic telescopes virtually guarantees that there will be somewhere on the globe suited for observing important astronomical phenomena.

\section{OBSERVING THE CHANGING UNIVERSE}

Much of modern astronomy research is devoted to things that are changing on time scales of minutes or days, and not millennia. The explosions of massive stars, asteroids passing close to the Earth, and new planets outside our Solar System are all examples of events that need to be investigated quickly. If these types of events are not studied in depth soon after their discovery, there may not be another chance, and the opportunity to learn more about them could be lost (see Figure 2).

Most of these interesting phenomena are found by giant telescopes that are regularly surveying the whole sky. Given that their job is to continually scan the whole sky, when these telescopes find something new they do not have time to look in detail. This is where robotic telescopes have a unique role to play. Robotic telescopes can be alerted to a new discovery and they can start observing it straight away. Making measurements quickly is often essential to understanding the science behind these exciting events. These observations and measurements help astronomers to understand the universe in a way that has never been possible before. 
Figure 2

Supernova SN2011fe in the distant Pinwheel galaxy. The supernova looks like a bright star from our own galaxy, but it is actually millions of times further away in a distant galaxy. It is the explosion of a star many times larger than the Sun when it reached the end of its life. The way its brightness changed with time was key to understanding this type of phenomenon and was only possible by using a network of robotic telescopes. This image was taken using Las Cumbres

Observatory's robotic telescope network.

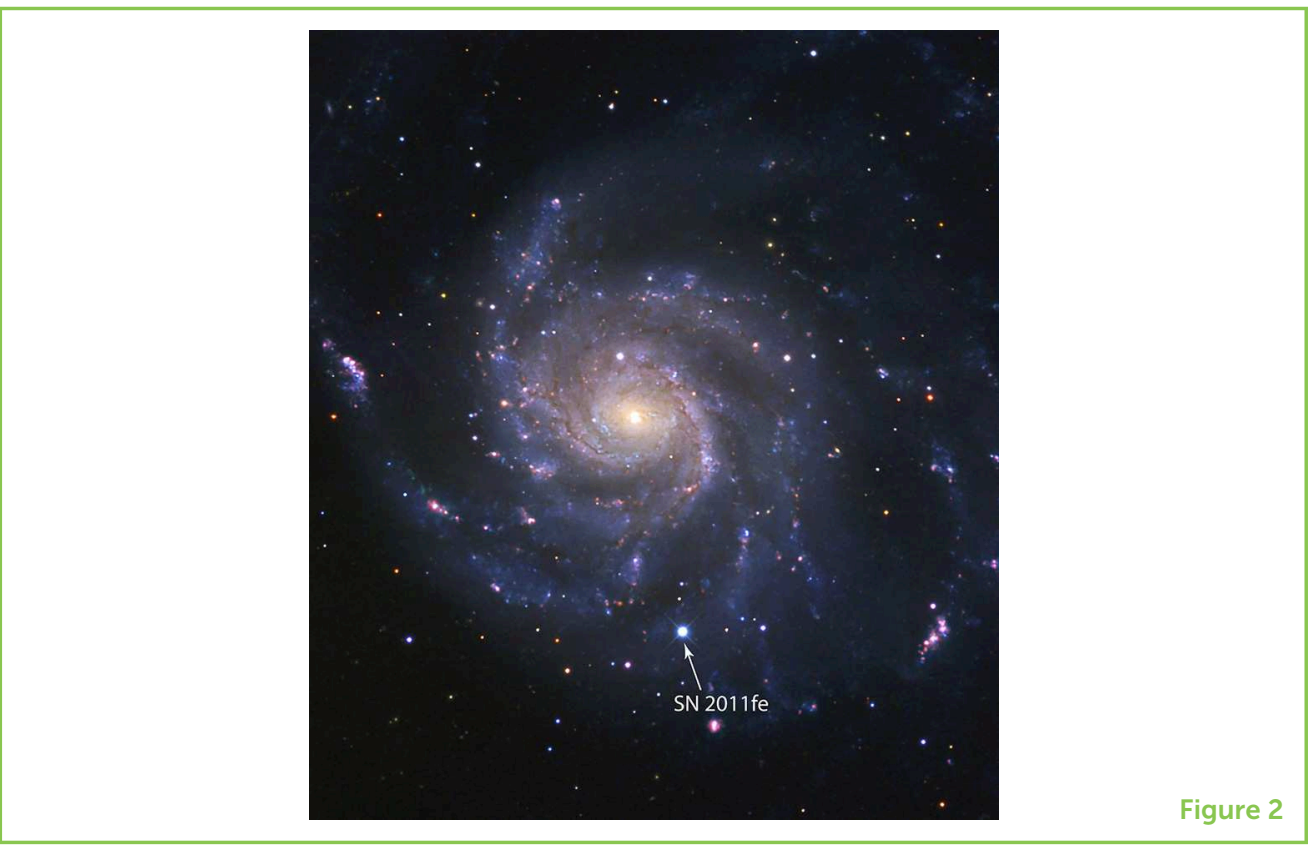

\section{EDUCATION}

Robotic telescopes also have a unique role to play in education. Students and teachers can now use some of the world's most technologically advanced and high quality professional observatories for their own investigations. With these online robotic observatories, students in schools can make discoveries and publish their research findings without ever leaving their classrooms.

The capabilities of telescopes are continually evolving and will only get better with time. Four hundred years ago, with a telescope the size of a small dog, Galileo revolutionized the way we view the Universe. Today, using robotic telescopes, professional and amateur astronomers, school students and their teachers can all change the way we view the Universe by making their own important discoveries.

\section{AUTHOR CONTRIBUTIONS}

The author confirms being the sole contributor of this work and has approved it for publication.

\section{FURTHER READING}

1. SkyNet. Available online at: https://skynet.unc.edu/

2. Las Cumbres Observatory. Available online at: https://lco.global

SUBMITTED: 19 February 2019; ACCEPTED: 18 June 2019;

PUBLISHED ONLINE: 04 July 2019. 
EDITED BY: Shane L. Larson, Northwestern University, United States

CITATION: Gomez E (2019) Why Put Telescopes at the Top of Mountains and Other Strange Places? Front. Young Minds 7:90. doi: 10.3389/frym.2019.00090

CONFLICT OF INTEREST STATEMENT: The author declares that the research was conducted in the absence of any commercial or financial relationships that could be construed as a potential conflict of interest.

COPYRIGHT @ 2019 Gomez. This is an open-access article distributed under the terms of the Creative Commons Attribution License (CC BY). The use, distribution or reproduction in other forums is permitted, provided the original author(s) and the copyright owner(s) are credited and that the original publication in this journal is cited, in accordance with accepted academic practice. No use, distribution or reproduction is permitted which does not comply with these terms.

\section{YOUNG REVIEWER}

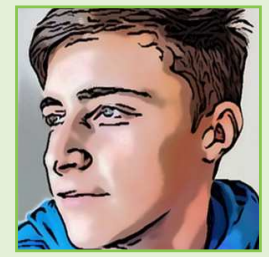

\section{GREG, AGE: 15}

$\mathrm{Hi}$, I am 15 years old and having a Transition Year (year out from school between exam courses) from home. My main interest is in physics but I am happy to read most science information that comes my way.

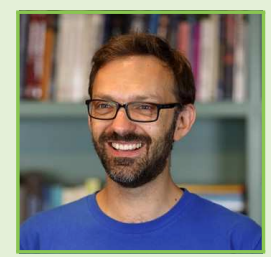

\section{AUTHOR}

\section{EDWARD GOMEZ}

I am an astronomer at Las Cumbres Observatory. I also spend a lot of my time writing computer code (using Python) to do research and use our robotic telescopes. I manage our global educational projects for school students, teachers, and the public using Las Cumbres Observatory to follow their own investigations and be inspired by space. I am also a member of the LCO Solar System research group, monitoring asteroids, and comets. *egomez@lco.global 